\title{
ChemComm
}

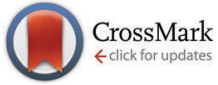

Cite this: Chem. Commun., 2015, 51,2863

Received 20th November 2014 Accepted 3rd January 2015

DOI: $10.1039 / c 4 c c 09277 e$

www.rsc.org/chemcomm

\section{Surfactant free superparamagnetic iron oxide nanoparticles for stable ferrofluids in physiological solutions $\dagger$}

\author{
K. Mandel, ${ }^{\star a}$ M. Straßer, ${ }^{a b}$ T. Granath, ${ }^{c}$ S. Dembski and G. Sextl ${ }^{a b}$
}

\begin{abstract}
A process is reported to obtain a nanoparticle sol from co-precipitated iron oxide particles without using any surfactant. The sol - a true ferrofluid - is not only stable over a wide range of $\mathrm{pH}$ but also in physiological solutions. This is a decisive step towards biomedical applications where nanoparticle agglomeration could so far only be prevented by using unwanted surfactants.
\end{abstract}

Using (superpara)magnetic nanoparticles in the field of medicine is a potentially very promising approach. For instance, tumour diagnosis in vivo could be carried out via a novel, direct imaging method called magnetic particle imaging ${ }^{1}$ and therapy might be possible via hyperthermia, i.e., the thermal destruction of tumour cells via inductive heating of magnetic nanoparticles. ${ }^{2}$ To render (superpara)magnetic nanoparticles applicable to these biomedical approaches, they need to meet two conditions:

For a proper use within the body, firstly, individual particles of a few nanometres in size in dispersion and no large agglomerates/ aggregates of particles are required. Secondly, the nanoparticle surfaces should be suitable for further attachment of biomolecules e.g. antibodies, necessary for a targeting of tumour cells. However, usually, the nanoparticle surfaces are covered with molecules from the synthesis process. These molecules might be often cytotoxic or prohibit further coverage of the nanoparticle surfaces with linkers.

To date, a huge amount of publications exists on the synthesis and functionalisation of (superpara)magnetic (iron oxide) nanoparticles (excellent reviews are available, see for instance ${ }^{3-6}$ ). However, only a minority of the researchers focus their research on the dispersion state of the nanoparticles. Those who consider this point, typically argue on the proper choice of surface active agents/surfactants/ surface modifying molecules to obtain a

\footnotetext{
${ }^{a}$ Fraunhofer Institute for Silicate Research ISC, Neunerplatz 2, 97082 Wuerzburg, Germany. E-mail: karl-sebastian.mandel@isc.fraunhofer.de

${ }^{b}$ Department of Chemical Technology of Materials Synthesis, University of Wuerzburg, Roentgenring 11, 97070 Wuerzburg, Germany

${ }^{c}$ Department Biology/Chemistry, University of Bremen, Bibliothekstraße 1, 28359 Bremen, Germany

† Electronic supplementary information (ESI) available. See DOI: 10.1039/ c4cc09277e
}

proper dispersion of nanoparticles in fluid $(=\mathrm{sol}) .^{7-22}$ This approach is rational and usually necessary, as nanoparticle dispersions can be either stabilised electrostatically, sterically or electro-sterically. ${ }^{23}$ In either case, this is generally achieved via anchoring molecules to the particle surfaces. However, for the envisaged in vivo application of nanoparticles, the draw-back is as described above. On the other hand, avoiding the use of surface stabilising molecules during the synthesis generally leads to agglomerates, which are also useless for the desired purpose.

Thus, it remains a crucial challenge to find a way to obtain a stable sol of individually dispersed nanoparticles at sufficiently high concentrations in physiological solutions and at variable $\mathrm{pH}$. Nanoparticles are needed that do not agglomerate at conditions usually encountered in the human body, without using any surface active agents/surfactants/surface modifying molecules.

Here we report about the development of such a particle system, comprised of superparamagnetic iron oxide nanoparticles, synthesised in an inexpensive and scalable way, forming highly concentrated, stable sols over a wide range of $\mathrm{pH}$ and in physiological solutions.

Superparamagnetic iron oxide nanoparticles (diameter $\sim 10 \mathrm{~nm}$ ) were synthesised via the well-established co-precipitation method: ${ }^{24}$ Typically $2.16 \mathrm{~g}$ ( $8 \mathrm{mmol}) \mathrm{FeCl}_{3} \cdot 6 \mathrm{H}_{2} \mathrm{O}$ and $0.795 \mathrm{~g}(4 \mathrm{mmol})$ $\mathrm{FeCl}_{2} \cdot 4 \mathrm{H}_{2} \mathrm{O}$ are dissolved in $100 \mathrm{ml}$ deionised water and precipitated with $5 \mathrm{ml}$ ammonia solution (28-30 wt\%) under stirring. This yielded agglomerates of the superparamagnetic iron oxide (magnetite) nanoparticles. A detailed characterisation of the chemistry, structure and magnetism of these particles was reported by us elsewhere. ${ }^{25-27}$

We reported on the dispersion of these nanoparticles to obtain an acidic, aqueous ferrofluid by adding diluted nitric acid $(20 \mathrm{ml}$, $0.5 \mathrm{M}$ ) to the agglomerates in an earlier work. ${ }^{25}$ However, such an acidic, electrostatically (positive surface charge from the protons) stabilised ferrofluid faces severe agglomeration as soon as the $\mathrm{pH}$ or the ionic strength of the solution is increased, as expected from the DLVO theory. ${ }^{28}$

A ferrofluid (=in a general definition: a sufficiently high concentration, typically $>20 \mathrm{mg} \mathrm{ml}^{-1}$, of individual nanoparticles in solution) stable over a wide range of $\mathrm{pH}$ and in physiological solutions (in solutions with high ionic strength) 
without using any surfactant can be obtained from the acidic ferrofluid by three elementary steps:

(1) Careful dilution steps; (2) addition of the right amount of tetraethoxy-silane (TEOS) to deposit silica on the particle surface and (3) a final centrifugation step where a specific fraction in the centrifuge tube is eventually selected.

In detail, the acidic ferrofluid was further diluted by a factor of 5 with deionised water before $5 \mathrm{ml}(22.5 \mathrm{mmol})$ of TEOS were carefully dropped into the stirred solution within 30 minutes. The wrong choice of dilution or amount of TEOS immediately leads to gelation within the acidic (aqueous) ferrofluid due to uncontrolled hydrolysis and condensation reactions. After an hour of stirring, the solution was further diluted by a factor of 2 and neutralised by adding sodium hydroxide solution (1 M) dropwise until a $\mathrm{pH}$ of 7-8 was reached. Without further dilution before neutralisation, again, gelation and agglomeration are immediately faced.

Then, the highly diluted solution is centrifuged for $180 \mathrm{~min}$ at $12000 \mathrm{rpm}$.

What is obtained in the centrifuge tube after centrifugation is a three phase system (see Fig. 5), in which the upper layer is a rather clear liquid and the bottom layer is a dense, gel-like sediment. The gelation of a certain portion of the particles in the fluid in the tube is induced by the centrifugation step and cannot be prohibited.

The middle layer/phase in the tube is a black fluid. This targeted layer is a concentrated sol of superparamagnetic iron oxide nanoparticles - a true aqueous ferrofluid.

All synthesis steps were repeated three times to prove the reproducibility of the approach.

The remarkable magnetic behaviour of the ferrofluid, resulting from the - for silica coated magnetite particles - good magnetisation of the particles (saturation magnetisation: $29 \mathrm{emu}^{-1}$ ) and the very high concentration of magnetic nanoparticles in the fluid (56 $\mathrm{mg}$ nanoparticles per $\mathrm{ml}$ fluid), is visualised in Fig. 1. The fluid in the vial is strongly attracted by a magnet. Image series Fig. 1 (images 1-6) demonstrates the stretching of a magnetic fluid droplet in a plastic vial, when a magnet is approached from the top.

The crucial condition for a proper ferrofluidic behaviour (Fig. 1) of superparamagnetic nanoparticles is that the nanoparticles are individually dispersed and do not agglomerate. The particles must undergo Brownian motion so that solidliquid separation in a magnetic field does not occur, but rather the whole fluid moves towards the magnet. ${ }^{29}$
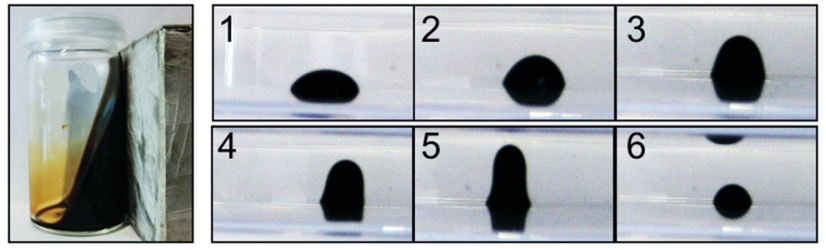

Fig. 1 Ferrofluidic properties of the synthesised nanoparticle sol. Lefthand-side image: in a vial next to a magnet; right-hand-side image series: for a single droplet. For the image series 1-6, a magnet was approached from the top.
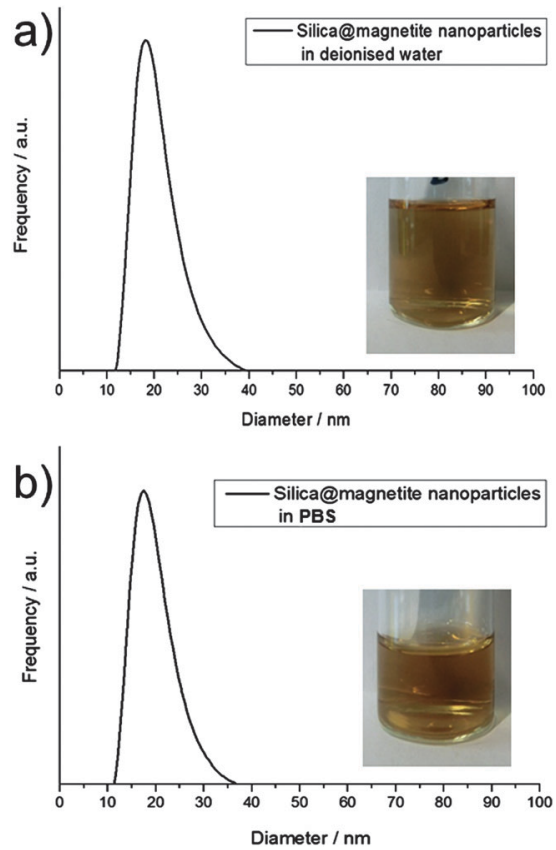

Fig. 2 Synthesised nanoparticles in deionised water (a) and in PBS (b) do not form agglomerates, as evident from the measured hydrodynamic diameter and the bright-clear appearance of a diluted ferrofluid.

These required conditions are met with the particle system reported in here.

Dynamic light scattering was used to quantify the particle size in dispersion. Fig. 2a shows the hydrodynamic diameter of the particles in solution, according to the laser diffraction results. The inset image shows a diluted sol; the bright-clear appearance is a strong empirical proof for a real nanodispersion (sol) that is agglomerate free. ${ }^{29}$

Subsequently, the stability of the nanoparticle dispersion in various other solutions was examined.

It turned out that a stable dispersion can also be retained in physiological solutions.

To achieve this, the ferrofluid was mixed (v/v $=50 / 50)$ with either a phosphine buffered solution (PBS), Dulbecco's Modified Eagle Medium (DMEM) or a fetal bovine serum (FBS) solution. A comparison of the hydrodynamic diameters of particles in PBS (Fig. 2b) with the particles in deionised water revealed that no agglomeration occurred, despite the high salt content. Moreover, the same bright-clear solution results from dilution as in Fig. 2a.

On top of that, the same stability was also observed for fluids in which the $\mathrm{pH}$ was varied between 2 and 10 via addition of droplets of $1 \mathrm{M}$ nitric acid and $1 \mathrm{M}$ sodium hydroxide to the ferrofluid.

A key-property for the remarkable dispersion-stability of the particles in solution is the nature of the silica coating.

Silica coating on magnetic nanoparticles, in principle, has been reported before. ${ }^{8,9,30-37}$ Recent interest in this coating arose, as it was found that a silica shell on iron oxide nanoparticles improved the biocompatibility of the particles, as cytotoxicity with such particle systems was significantly reduced. ${ }^{35-37}$ In an excellent 
work by R. K. Singh et al., ${ }^{37}$ the necessity (with respect to biomedical applications of such particles) to not only achieve silica coated iron oxide nanoparticles, but also yield an agglomerate-free, aqueous particle dispersion, was emphasized yet as well. It was observed by the group that already a thin silica coating helps to obtain particle dispersions in water at neutral $\mathrm{pH}$ that do not sediment within $30 \mathrm{~min}$ due to agglomeration. The reason is that a silica surface possesses a sufficiently negative zeta potential at $\mathrm{pH} 7$, maintaining repulsion between particles. However, such particles might agglomerate when salt is added to the water, as known from the DLVO theory. ${ }^{28}$

The remarkable ferrofluidic properties over a wide range of $\mathrm{pH}$ and in salt-containing solutions for silica coated, but otherwise surfactant-free particles, as presented in our work here, has not been reported in that way before. The reason might be that even if a thin silica coating onto individual particles is achieved agglomeratefree, this does not yield a sol which is indifferent to a $\mathrm{pH}$ change and salt to such a great extent as such particles would behave like typical Stöber silica particles, which are not stable as a sol in a changing $\mathrm{pH}$ environment or at a high salt content. ${ }^{38}$ As stated, this is, as according to the DLVO theory, agglomeration occurs as soon as the $\mathrm{pH}$ is close to the iso-electric point or as soon as the salt content is high enough to shrink the electric double layer around the particles, thus destroying their repulsion. ${ }^{28}$

It is therefore assumed that the remarkable stability observed for the particles synthesised in this work is due to a distinct type of silica surface coating.

First of all, Fourier-transformed infrared spectroscopy (FTIR) proves that both, silica as well as iron oxide is what the ferrofluid is composed of (additionally, FTIR bands from some residuals from the synthesis, namely nitrate and organic from TEOS, are found), as can be seen in Fig. 3. (N.B.: with respect to biomedical applications, potential residual ethanol could be easily boiled out from the sol.)

Transmission electron microscopy (TEM) of the particles, as depicted in Fig. 4, reveals the interconnection of silica and iron oxide: silica is always adjunct to the iron oxide nanoparticles. More precisely, the iron oxide nanoparticles (dark spots) are all surrounded by a brighter fringe, which is composed of silica. However, the silica cover is rather "imperfect"/"patchy" and not completely enclosing the iron oxide $\left(\mathrm{Fe}_{3} \mathrm{O}_{4}\right)$ nanoparticles.

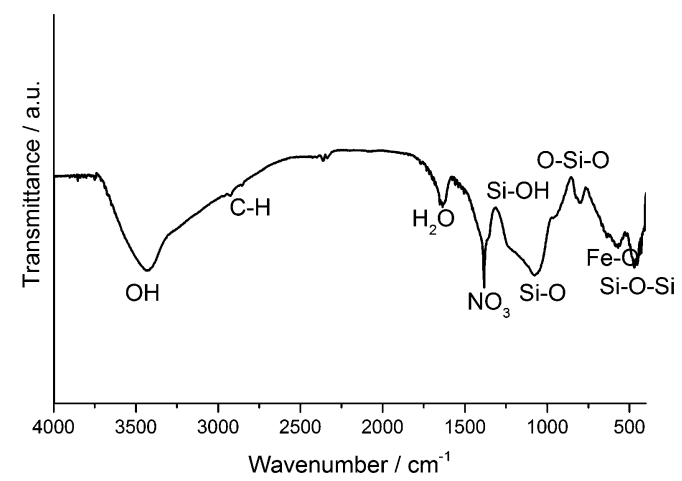

Fig. 3 FTIR reveals that the sol is composed of silica and iron oxide.

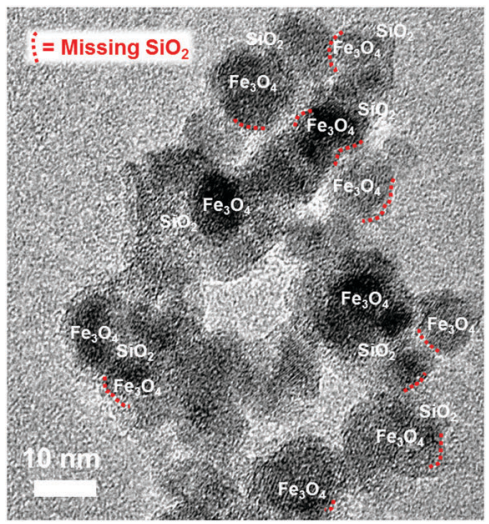

Fig. 4 TEM shows silica coating on magnetite nanoparticles $\left(\mathrm{Fe}_{3} \mathrm{O}_{4}\right)$, however the coating is "imperfect".

As described in the experimental section, besides these particles, forming the ferrofluid, which are found in the middle-layer phase of the centrifuge tube, there was also an upper, clearer layer and a gel-like bottom sediment observed in the tube. Whereas in the upper layer phase only residual and unreacted silica remains, the bottom layer phase (the gel-like layer) consists of iron oxide nanoparticles enclosed in network of condensed silica (TEM, Fig. S1, ESI $\dagger$ ). Fig. 5 shows a scheme, summarising what is found in the three layers within the tube. The following can be concluded: to obtain a ferrofluid with the remarkable properties as described in this work, iron oxide nanoparticles with a "patchy-layer" of $\mathrm{SiO}_{2}$ need to be selected. This selection can be conveniently done by "filtering out" the "good" fraction of particles via picking the middle layer phase after centrifugation. This middle layer phase can be easily identified as only this layer acts as a vital magnetic fluid when a magnet is approached.

More surface-information on the remarkable nature of "the particles from the centrifuge tube's middle-layer", especially in terms of surface charge, which depends on the actual surface chemistry, can be obtained from zeta potential measurements. Fig. 6 shows the zeta potential curve of the imperfectly silica coated iron oxide (magnetite) nanoparticles as function of the $\mathrm{pH}$ in comparison to the zeta potential curves of uncoated magnetite nanoparticles and pure silica nanoparticles.

The zeta potential curve measured for the particles synthesised in this work, somewhat lies in-between the values for pure silica and magnetite. As no other surface-modifying molecules can come into question to yield this result, the curve can only be interpreted by assuming particles that are not fully coated

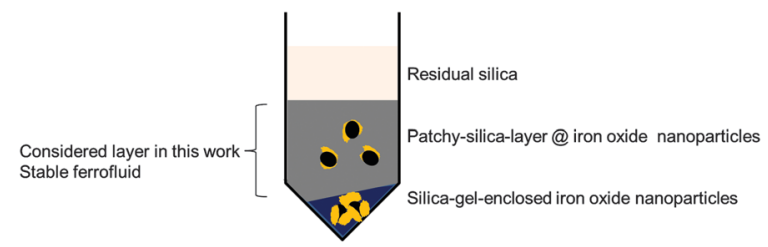

Fig. 5 Scheme of the three layers in the centrifuge tube obtained after silica coating synthesis. The middle layer needs to be selected to obtain the ferrofluid with the remarkable properties as discussed in this work. 


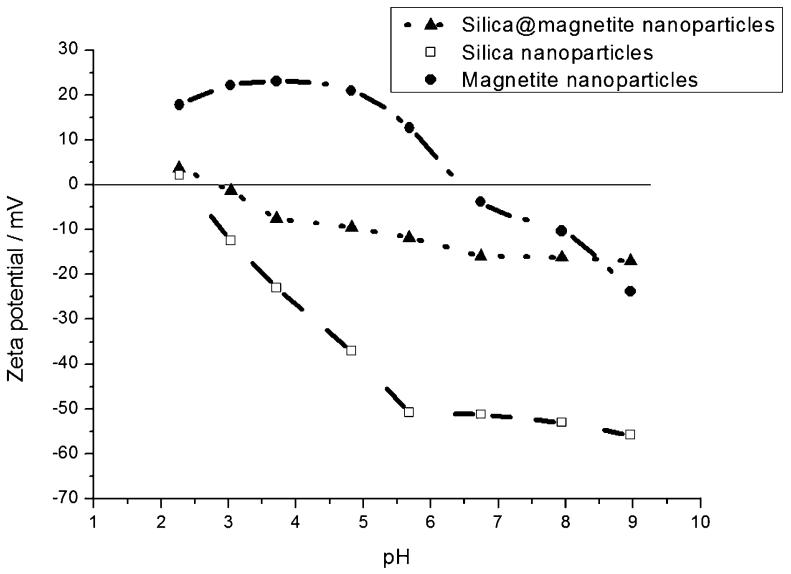

Fig. 6 Zeta potential curve values of the magnetite nanoparticles with "imperfect" silica coating lie in-between the values obtained for pure magnetite or silica nanoparticles.

with silica, i.e., patchy particles, that adopt a kind of "twofaced" behaviour. As via the method to determine the zeta potential, the surface charge of the particles in an aqueous solution is recorded as function of the $\mathrm{pH}$ in solution, the mixture of positive and negative surface parts results in a somewhat "in-between" zeta potential curve compared to the positive magnetite surface and the negative silica surface (within the considered $\mathrm{pH}$ range). This is because the method is actually based on recording the particle movement in an electric field and translating this to surface charge values. When there is no distinct positive or negative surface charge prevailing, such "twofaced"/"patchy" particles move slower, which appears as a lowered zeta potential in the measurement result.

This might be an explanation why the nanoparticle sols are so unexpectedly stable under a wide range of $\mathrm{pH}$ and under conditions in a solution of high ionic strength: as there is no uniform surface chemistry but rather a differently charged surface under different conditions (compare the zeta potential of pure magnetite nanoparticles versus pure silica nanoparticles), the classical ways of destroying the stability of dispersed nanoparticles ( $\mathrm{pH}$ change; addition of salt) do not cause agglomeration in this system.

The system reported in here is somewhat like an inorganic material pendant to a zwitterionic polymer.

Thus, an "imperfect"/"patchy" silica coating is the key to a stable sol of superparamagnetic nanoparticles, which stays stable under conditions prevailing in the human body.

Although silica coating of magnetic nanoparticles has been reported quite often to date, it was not yet considered as an approach towards obtaining a stable dispersion (sol/ferrofluid) of superparamagnetic iron oxide nanoparticles without any surfactant at various conditions. With the reported process to create an "imperfect"/"patchy" silica coating on iron oxide nanoparticles, agglomerate-free sols over a wide range of $\mathrm{pH}$ and in physiological solutions are obtained. Such patchy particles, only consisting of iron oxide and silica, might be a potentially very helpful building block in the task of developing and ultimately establishing the application of (superpara)magnetic nanoparticles in tumour diagnostics and therapy for the medical treatment of tomorrow.

\section{Notes and references}

1 B. Gleich and J. Weizenecker, Nature, 2005, 435, 1214.

2 I. Hilger, R. Hergt and W. A. Kaiser, J. Magn. Magn. Mater., 2005, 293, 314.

3 R. G. Chaudhuri and S. Paria, Chem. Rev., 2012, 112, 2373.

4 A.-H. Lu, E. L. Salabas and F. Schüth, Angew. Chem., Int. Ed., 2007, 46, 1222.

5 M. Mahmoudi, S. Sant, B. Wang, S. Laurent and T. Sen, Adv. Drug Delivery Rev., 2011, 63, 24.

6 S. Laurent, D. Forge, M. Port, A. Roch, C. Robic, L. Vander Elst and R. N. Muller, Chem. Rev., 2008, 108, 2064.

7 S. Sun and H. Zeng, J. Am. Chem. Soc., 2002, 124, 8204.

8 R.-Y. Hong, J.-H. Li, S.-Z. Zhang, H.-Z. Li, Y. Zheng, J.-m. Ding and D.-G. Wei, Appl. Surf. Sci., 2009, 255, 3485.

9 Y. Leng, K. Sato, J.-G. Li, T. Ishigaki, M. Iijima, H. Kamiya and T. Yoshida, Powder Technol., 2009, 196, 80.

10 X. Q. Xu, H. Shen, J. R. Xu, J. Xu, X. J. Li and X. M. Xiong, Appl. Surf. Sci., 2005, 252, 494.

11 S. García-Jimeno and J. Estelrich, Colloids Surf., A, 2013, 420, 74.

12 H. T. Chan, Y. Y. Do, P. L. Huang, P. L. Chien, T. S. Chan, R. S. Liu, C. Y. Huang, S. Y. Yang and H. E. Horng, J. Magn. Magn. Mater., 2006, 304, e415-e417.

13 C.-L. Lin, C.-F. Lee and W.-Y. Chiu, J. Colloid Interface Sci., 2005, 291, 411.

14 A. Bocanegra-Diaz, N. D. S. Mohallem, M. A. Novak and R. D. Sinisterra, J. Magn. Magn. Mater., 2004, 272-276, 2395.

15 M. T. López-López, J. D. G. Durán, A. V. Delgado and F. GonzálezCaballero, J. Colloid Interface Sci., 2005, 291, 144.

16 V. Ervithayasuporn and Y. Kawakami, J. Colloid Interface Sci., 2009, 332, 389.

17 D. K. Kim, Y. Zhang, W. Voit, K. V. Rao and M. Muhammed, J. Magn. Magn. Mater., 2001, 225, 30.

18 E. H. Kim, H. S. Lee, B. K. Kwak and B.-K. Kim, J. Magn. Magn. Mater., 2005, 289, 328.

19 D. Maity and D. C. Agrawal, J. Magn. Magn. Mater., 2007, 308, 46.

20 R. Y. Hong, B. Feng, L. L. Chen, G. H. Liu, H. Z. Li, Y. Zheng and D. G. Wei, Biochem. Eng. J., 2008, 42, 290.

21 L. Vékás, D. Bica and M. V. Avdeev, China Particuol., 2007, 5, 43.

22 S. Genc and B. Derin, Curr. Opin. Chem. Eng., 2014, 3, 118.

23 D. J. Shaw, Introduction to Colloid and Surface Chemistry, Butterworth-Heinemann, Oxford, 1992.

24 R. Massart, IEEE Trans. Magn., 1981, 17, 1247.

25 K. Mandel, F. Hutter, C. Gellermann and G. Sextl, Colloids Surf., A, 2011, 390, 173.

26 K. Mandel, W. Szczerba, A. F. Thünemann, H. Riesemeier, M. Girod and G. Sextl, J. Nanopart. Res., 2012, 14, 1066.

27 K. Mandel, F. Hutter, C. Gellermann and G. Sextl, J. Magn. Magn. Mater., 2013, 331, 269.

28 W. B. Russel, D. A. Saville and W. R. Schowalter, Colloidal dispersions, Cambridge University Press, New York, 1991.

29 K. Mandel and F. Hutter, Nano Today, 2012, 7, 485.

30 M. Tadić, V. Kusigerski, D. Marković, M. Panjan, I. MiloŠević and V. Spasojević, J. Alloys Compd., 2012, 525, 28.

31 Y. Sun, L. Duan, Z. Guo, Y. DuanMu, M. Ma, L. Xu, Y. Zhang and N. Gu, J. Magn. Magn. Mater., 2005, 285, 65.

32 H. Kazemzadeh, A. Ataie and F. Rashchi, J. Supercond. Novel Magn., 2012, 25, 2803.

33 Y.-H. Deng, C.-C. Wang, J.-H. Hu, W.-L. Yang and S.-K. Fu, Colloids Surf., A, 2005, 262, 87.

34 Y. Lu, Y. Yin, B. T. Mayers and Y. Xia, Nano Lett., 2002, 2, 183.

35 M. Abbas, S. R. Torati, C. S. Lee, C. Rinaldi and C. Kim, J. Nanomed. Nanotechnol., 2014, 5, 244.

36 L. Khanna and N. K. Verma, Mater. Lett., 2014, 128, 376.

37 R. K. Singh, T. H. Kim, K. D. Patel, J. C. Knowles and H. W. Kim, J. Biomed. Mater. Res., Part A, 2012, 100A, 1734.

38 T. Oertel, F. Hutter, U. Helbig and G. Sextl, Cem. Concr. Res., 2014, 58, 131. 